\title{
Clase pública de un estudio de clases de estadística: Una instancia de cambio de creencias en los profesores
}

\author{
Public Class of a Statistics Lesson Study: An Instance to Change Teachers' Beliefs
}

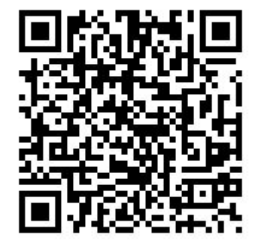

\author{
Raimundo Olfos' \\ Pontificia Universidad Católica de Valparaíso \\ Valparaíso, Chile \\ raimundo.olfos@pucv.cl \\ Soledad Estrella \\ Pontificia Universidad Católica de Valparaíso \\ Valparaíso, Chile \\ soledad.estrella@pucv.cl \\ Sergio Morales $^{3}$ \\ Pontificia Universidad Católica de Valparaíso \\ Valparaíso, Chile \\ sergio.morales.candia@gmail.com
}

Recibido 11 de octubre de 2014 • Corregido 30 de julio de 2015 • Aceptado 14 de agosto de 2015

\footnotetext{
1 Profesor de matemáticas. Doctor en Filosofía por la Universidad de Gales, Reino Unido. Posdoctorado en Pensamiento Matemático en la Universidad de Londres. Miembro claustro doctorado en Didáctica de la Matemática y Profesor del Departamento de Matemáticas de la Pontificia Universidad Católica de Valparaíso. Investigador del Centro de Investigación Avanzada en Educación del consorcio entre Universidad de Chile, Universidad de Concepción y Pontificia Universidad Católica de Valparaíso. Especialista Internacional en Estudio de Clases para la APEC. Autor de variados libros y de artículos en revistas de corriente principal. Investigador responsable de proyectos financiados por el Ministerio de Educación de Chile, y por la Comisión Nacional de Investigación en Ciencia y Tecnología (CONICYT).

2 Profesora de Matemática y Computación. Magister en Didáctica de la Matemática. Doctor en Didáctica de la Matemática por la Pontificia Universidad Católica de Valparaíso. Miembro claustro doctorado en Didáctica de la Matemática y Profesor del Departamento de Matemáticas de la Pontificia Universidad Católica de Valparaíso. Especialista en Didáctica de la Estadística, en formación inicial y continua de profesores, y en Estudio de Clases. Autora de variados artículos en revistas de corriente principal. Investigadora responsable de proyectos financiados por la Comisión Nacional de Investigación en Ciencia y Tecnología (CONICYT).

${ }^{3}$ Profesor de Matemáticas de Enseñanza Media. Magister en Didáctica de la Matemática. Profesor líder en Estudio de Clases del Liceo Comercial Francisco Araya Bennett de Valparaíso. Asistente de Investigación Departamento de Matemáticas de la Pontificia Universidad Católica de Valparaíso.
} 
doi: http://dx.doi.org/10.15359/ree.19-3.21

URL: http://www.una.ac.cr/educare

CORREO: educare@una.cr

Resumen. Se investiga el impacto de la implementación de una lección de estadística en las creencias del profesorado que observa la lección. Bajo un diseño cuasi-experimental participan del estudio 18 docentes y 7 estudiantes de docencia, quienes responden un cuestionario validado, con 6 proposiciones bajo una escala Likert. El cuestionario de creencias sobre cómo enseñar estadística se aplicó individualmente a cada docente cuando llega al salón donde se realiza la clase pública, y al terminar la misma, es respondido como postest. La clase observada tuvo una duración de alrededor de 90 minutos. Se usó la prueba no paramétrica de Wilcoxon para comparar las medianas entre ambas aplicaciones. Los resultados dan cuenta de leves diferencias en "cómo enseñar estadística", sin llegar a ser significativa. Esta investigación invita a continuar la exploración y comprensión del cambio de creencias a partir de una clase pública de Estudio de Clases como método de formación continua.

Palabras claves: estudio de clase, clase pública, enseñanza de la estadística, creencias.

Abstract. This paper presents a research on the impact of the implementation of a Statistics Lesson from the point of view of the beliefs of the teachers who observed the lesson. Eighteen teachers and seven future teachers participated in this quasi-experimental design and responded to a validated questionnaire with six propositions under a Likert scale. The questionnaire of beliefs on how to teach Statistics was applied individually to each teacher when they came to the room where public class was being delivered and it was responded as a posttest. The class observed lasted around 90 minutes. The non-parametric Wilcoxon test was used to compare the averages between the two applications. The results show slight differences, which do not become significant, on "how to teach Statistics". This research is an invitation to a further exploration and understanding of the changes of beliefs based on a public class of a lesson study as a continuous training method.

Keywords. Lesson study, public class, Statistics teaching, beliefs.

\section{Introducción}

La razón de ser de la enseñanza de la estadística a nivel escolar es la alfabetización estadística necesaria para que en el futuro el estudiantado pueda ser competente en la sociedad de la información. Su inclusión en el currículo pretende que la población estudiantil lea, registre, analice y realice predicciones a partir de datos presentes en distintos tipos de dispositivos (Ministerio de Educación [MINEDUC], 2012). Nos encontramos en un contexto creciente de requerimientos de alfabetización estadística y modificaciones en el currículo de la educación primaria; lo cual no se condice con la velocidad en la actualización del profesorado para asumir estos cambios.

Al respecto, la bibliografía internacional en el área nos permite saber que el personal docente de primaria presenta una débil formación en educación estadística (Shaughnessy, 2007; Franklin y Mewborn, 2006). La evidencia indica que los docentes y las docentes confunden los propósitos de la enseñanza de la estadística, identificándolos con el aprendizaje de reglas para calcular medidas de tendencia y técnicas para representar datos por medio de tablas y gráficos (Araneda, Del Pino, Estrella, Icaza y San Martín, 2011; Ben-Zvi y Sharett-Amir, 2005; Del Pino y Estrella, 2012; Garfield y Ben-Zvi, 2007). 
Emerge, entonces, la necesidad de considerar la actualización del profesorado de estadística, dentro del mapa de desafíos para la implementación y despliegue de los requerimientos curriculares. En este mapa, se debe considerar el gran tamaño de la población de docentes en ejercicio, y los distintos dispositivos presentes para desplegar acciones de formación continua. Parte de nuestra inquietud surge por considerar que la proporción del profesorado chileno de educación primaria que participa en educación continua tradicional formal (programas que emiten una certificación) es baja en relación con la cantidad de docentes con formación en estadística y en su didáctica que demandan los sistemas educativos. Por ejemplo, en un trabajo anterior dimos cuenta de que en Chile se necesitarían 30 años de cursos de formación continua tradicional formal para cubrir la población docente que requiere actualización en matemática (Olfos, 2010). Emerge entonces la necesidad de revisar y seleccionar una estrategia de formación continua de docentes que permita un despliegue mayor, sea viable e influya positivamente en estas demandas de formación.

El estudio de clases es un método de formación continua y de desarrollo profesional docente basado en la colaboración, que ha sido citado como un factor clave en la mejora de la enseñanza de las matemáticas en los sistemas en los cuales ha sido implementado (Fernández y Yoshida, 2004; Stigler y Hiebert, 1999). El estudio de clases es la principal estrategia de desarrollo profesional en las escuelas primarias japonesas, siendo implementado en muchos de los países miembros de la Cooperación Económica de Asia Pacífico, APEC (Fang, Lee, y Sharifath, 2009). Perry y Lewis (2009) describen el proceso como un ciclo en que los profesores y profesoras trabajan juntos para

a) formular objetivos en colaboración para el aprendizaje y el desarrollo a largo plazo de la población estudiantil;

b) planear una "lección a investigar" diseñada para llevar a la práctica tales objetivos;

c) implementar la lección gestionada por un miembro del equipo, reuniendo evidencia sobre el aprendizaje y desarrollo del estudiantado; y

d) reflexionar y discutir sobre las evidencias reunidas durante la clase, utilizándolas para mejorar la lección, la unidad de aprendizaje y la enseñanza.

Con respecto a las evidencias sobre estudio de clases, se cuenta con información que reconoce su impacto y contribución en la formación docente. Así, en los Estados Unidos, se ha valorado el estudio de clases para el mejoramiento de la formación docente (Lewis, Perry, Hurd, y O'Connell, 2006; Rock y Wilson, 2005). Hart, Alston y Murata (2011) editan un libro que aborda la implementación del estudio de clases en 16 lugares diferentes alrededor del mundo, mostrando las experiencias en investigación y en la práctica del estudio de clases. A su vez, las conclusiones de un trabajo investigativo sobre estudio de clases, de dos grupos de profesores de primaria en Singapur, destacan que "the knowledge contribution from the experienced teachers and subject specialists was significant in developing the pedagogical content knowledge in the community of practice" [la contribución al conocimiento de docentes especialistas y con 
doi: http://dx.doi.org/10.15359/ree.19-3.21

URL: http://www.una.ac.cr/educare

CORREO: educare@una.cr

experiencia en la materia fue significativo en el desarrollo del conocimiento pedagógico del contenido en la comunidad de práctica] (Fang y Lee, 2010, p. 3).

Por su parte, también grupos de docentes de escuelas de Singapur informan que el estudio de clases tiene un enorme potencial en las concepciones docentes y enfoques de aprendizaje (Yoong, 2011). Según Fang et al. (2009), las personas participantes en su estudio "they had developed a well-blended form of pedagogical content knowledge that is directly applicable to improve pupil's deep understanding of these topics" [han desarrollado una forma de conocimiento pedagógico del contenido que es aplicable directamente a mejorar la comprensión profunda del alumno en esos temas] (p. 126).

La evidencia da cuenta, entonces, de que este método influye en el conocimiento y concepciones que el personal docente presenta sobre la enseñanza y el aprendizaje. Estos hallazgos se vinculan con una línea que estudia las creencias del profesorado acerca de la enseñanza y el aprendizaje de la matemática (Felbrich, Kaiser y Schmotz, 2012; Pehkonen, 2004). En esta línea, existen escasos trabajos en el ámbito de la educación estadística (Zieffler, Park, Garfield y DelMas, 2012; Hassad, 2011; Estrada, Batanero y Lancaster, 2011).

Pehkonen (2004) distingue tres líneas de investigación en el estudio de las creencias en educación matemática: las creencias acerca de la matemática como disciplina, aprendizaje de la matemática y resolución de problemas, y enseñanza de la matemática en general. Para Handal (2003) existe consenso en los investigadores e investigadoras para estudiar ¿qué es matemática?, ¿cómo se enseña y aprende matemática? y ¿cómo la enseñanza y el aprendizaje de la matemática podrían mejorar?

Las creencias del profesorado regulan las prácticas docentes en el aula (Pehkonen, 2004) y aparecen como filtros a través de los cuales los profesores y las profesoras toman decisiones sobre la enseñanza, desempeñando un papel crucial en las prácticas en el aula (Felbrich et al., 2012; Furtak y Alonzo, 2010), incluso, a veces con mayor peso que el conocimiento pedagógico y las orientaciones curriculares. En la literatura e investigaciones sobre procesos de cambio e implementación de reformas curriculares, el rol de las creencias resulta crucial para favorecer o aplazar los procesos de cambio, dado su carácter conservador y relación dialéctica con las prácticas (Fullan y Stiegelbauer, 1997; Handal, 2003; Furtak y Alonzo, 2010; Pehkonen, 2004).

El propio sistema escolar aparece como un vehículo para transmitir y perpetuar creencias de orientación tradicional. En muchos países, el personal docente tiende a ser práctico, intuitivo e individualista en la toma de decisiones curriculares; y frente a la multiplicidad de eventos en el aula, busca movilizar sus creencias más que su conocimiento. Es así que el profesorado va generando sus propias creencias sobre cómo enseñar, las cuales son perpetuadas en sus prácticas (Handal, 2003). Si bien en las investigaciones el profesorado afirma que su enseñanza está orientada por sus creencias, también señala factores del contexto escolar (enfoque 
tradicional del sistema, administración escolar, padres, madres, estilo tradicionales de los alumnos, evaluaciones externas) que obstaculizan la implementación de sus creencias ideales y la innovación en la enseñanza de la matemática (Handal, 2003).

Como las creencias del personal docente acerca de la matemática influyen en sus prácticas de enseñanza, es posible suponer que tienen cierto grado de influencia en el aprendizaje del alumnado (Staub y Stern, 2002; Yates, 2006). Estudios mencionados por Felbrich et al. (2012) atribuyen un rol relevante a las creencias docentes en los resultados de rendimiento de los alumnos y alumnas en matemática.

La evidencia indica que las creencias son difíciles de cambiar, inclusive si se confrontan con pruebas contradictorias, comportándose a menudo resilientemente. Hart y Carriere (2011) señalan que uno de los objetivos del aprendizaje profesional no es solo proporcionar a al profesorado de nuevos marcos para usar mientras planifican experiencias de aprendizaje, sino también desafiar efectivamente las creencias fundamentales que conducen su toma de decisiones. Knapp Bomer y Moore (2011) reportan que la clase pública del estudio de clases proporciona un terreno fértil al personal docente para explicar sus creencias acerca de la enseñanza y para probar esas creencias en una clase pública.

En síntesis, la literatura provee evidencia de una relación dialéctica entre creencias y práctica. A su vez, existen pruebas de una mantención y perpetuación de estas en la práctica, con un enfoque del tipo tradicional, facilitado por componentes que son percibidos como obstáculos para la innovación en la enseñanza. Finalmente, se establece que las creencias desempeñan un papel crucial en la puesta en práctica del conocimiento del personal docente en las situaciones de aula.

El presente estudio se desarrolla en una modalidad particular del estudio de clases, la cual se denomina "clase pública" (Isoda y Olfos, 2009). Una clase pública se caracteriza por ser abierta a un público - usualmente constituido por docentes o estudiantes de pedagogía- que no participa en la preparación de la lección, pero acude con el fin de observar formas alternativas para conducir clases. En el proceso de observación, las personas espectadoras reflexionan sobre la propuesta de clase plasmada en una planificación, así como sobre la gestión de esta, considerando la interacción entre estudiantes y docente, además de los materiales utilizados.

Tanto el plan de la clase, como la lección observada y el debate que se implementa inmediatamente después, constituyen instancias que influyen en las creencias del profesorado acerca de cómo conducir la clase y qué aspectos privilegiar en esta. En efecto, el plan de la lección demuestra la calidad de la tarea propuesta al grupo de estudiantes, su nivel de demanda cognitiva, la elección de las variables didácticas y la organización de los momentos de la clase. El debate implementado después de impartir la lección también es portador de elementos descriptivos de las creencias, posibilitando su acceso a estas y permitiendo triangular la información puesta en juego en la lección (Olfos, Estrella y Morales, 2014). 
doi: http://dx.doi.org/10.15359/ree.19-3.21

URL: http://www.una.ac.cr/educare

CORREO: educare@una.cr

El presente estudio se sostiene bajo el supuesto de que las prácticas de enseñanza reciben una fuerte influencia de las creencias del personal docente y de que estas se modifican a partir de la reflexión sobre la práctica, en la práctica y de manera colaborativa; constituyéndose la clase pública en una potente herramienta para impulsar el proceso de cambio en las creencias docentes.

El objetivo del presente artículo es analizar el cambio en las creencias sobre enseñanza de la estadística de un grupo de docentes participantes de una clase pública.

\section{Método}

Este trabajo corresponde a un estudio exploratorio de implementación de una clase pública, considerando como variables las creencias que las personas participantes de esta clase reportaron antes y después de su observación. De esta manera, la unidad de análisis son docentes observadores de una clase pública, en quienes se estudian sus creencias acerca de la enseñanza de la estadística.

El diseño corresponde a un estudio cuasi-experimental, de tipo cuantitativo, con un muestreo no probabilístico, conformado por participantes voluntarios y voluntariasde una población de docentes que imparte clases de matemática con contenido estadístico. Se convocó a las personas participantes de manera abierta a una clase pública. Entre quienes observaron la clase pública, en la investigación se selecciona a las personas que respondieron el cuestionario antes y después de participar en la clase. Este estudio se realiza con 25 docentes o estudiantes de docencia de educación primaria: 7 sin experiencia y 18 con nueve años de experiencia en promedio.

\section{Tratamiento}

Las personas participantes observaron el desarrollo de una clase pública para un quinto grado. Esta lección fue preparada bajo la modalidad del estudio de clases en una sesión semanal durante dos meses. La figura 1 muestra el ciclo de este estudio de clases.

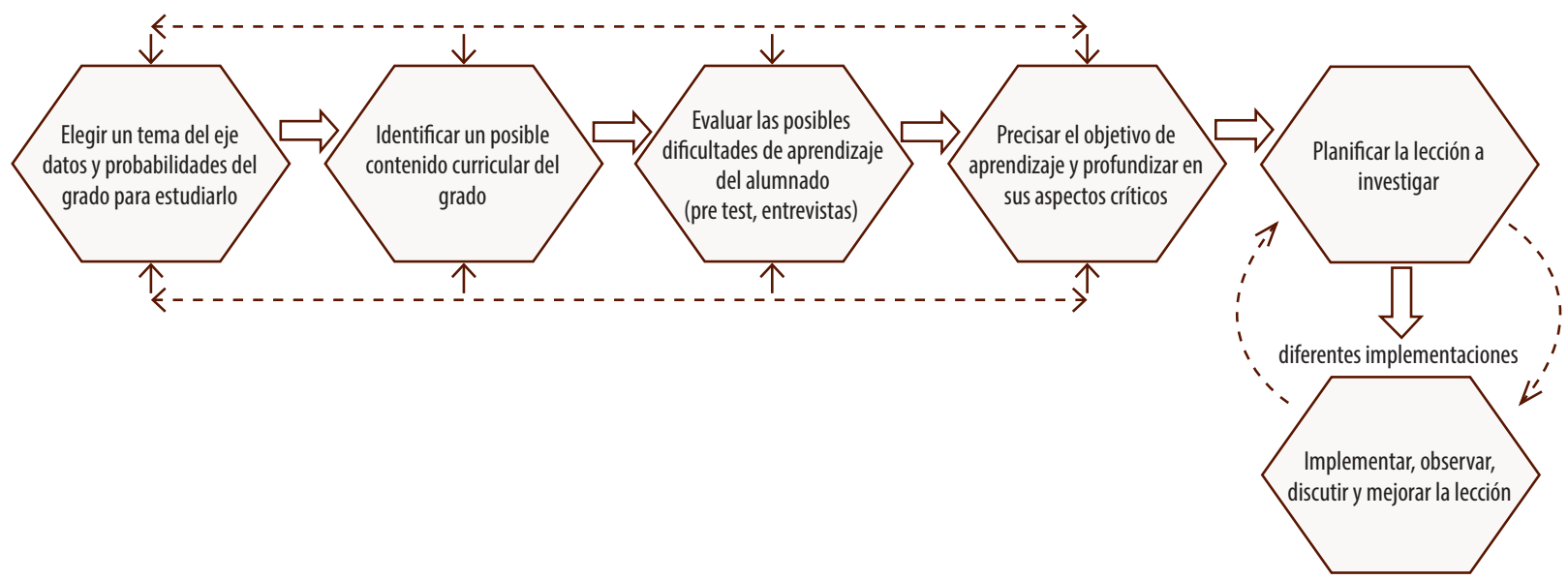

Figura 1. El ciclo de estudio de clases implementado. 
La preparación fue llevada a cabo por tres docentes de aula y tres personas investigadoras. Los objetivos de aprendizaje correspondieron al uso de representaciones de datos en estadística y la toma de decisiones en el análisis de datos, en un caso asociado a una noticia del tsunami que afectó a Chile en febrero del 2010 (Estrella, Olfos y Morales, 2014). Como parte del proceso, el grupo de estudiantes comienza con la exploración y termina con la presentación de sus productos a la clase. Se ponen en juego habilidades de modelación, que incluyen la capacidad de identificar preguntas relevantes, las variables, las relaciones en un contexto real, traducir e interpretar y validar la solución del problema con respecto al escenario planteado. Un profesor estuvo a cargo de implementar la lección con un curso de 40 estudiantes, en un tiempo de 90 minutos. El Anexo A muestra el plan de la lección entregado al inicio de la clase pública a las personas observadoras. Se describe a continuación la clase realizada.

El profesor comenzó la lección familiarizando al grupo de estudiantes con el tsunami y su efecto, luego planteó el desafío de "Extraer y organizar la información para comunicar a las ciudades que se vieron afectadas y podrían verse afectadas por los tsunamis". El profesor planteó al grupo de estudiantes el problema, diciendo "encontré esta foto en un periódico sobre el desastre natural de 2010, tiene mucha información, así que les solicito que organicen la información de forma más simple. El material que ustedes construyan lo enviaremos a las zonas afectadas para ser difundido y pueda salvar vidas".

El Anexo B muestra la lámina entregada a cada estudiante y a las personas participantes de la clase pública. Las preguntas usadas por el profesor para gestionar el momento de la discusión fueron: ¿es predecible el número de olas por ciudad?, ¿es predecible el tiempo que transcurre entre una ola y otra?, ¿es posible salvar vidas con esta información? Estaba considerada en la planificación la necesidad de enfrentar algunos mitos que habían costado vidas en el tsunami ocurrido en Chile, a saber, la creencia errónea de que "un tsunami es una ola grande" y que "existe un intervalo de tiempo fijo entre olas".

Durante la lección, el estudiantado fue capaz de hacer gráficos y tablas de distintos tipos. La figura 2 muestra producciones de diferentes estudiantes.

La clase se llevó a cabo en el tiempo previsto de 90 minutos. Luego tres panelistas expertos y el profesor reflexionaron y discutieron sobre la clase observada. Aunque el profesor declaró que la lección fluyó, él esperaba que el grupo de estudiantes produjeran más representaciones de datos; sin embargo, se mostró satisfecho y motivado, sobre todo porque los niños y niñas habían disfrutado del desafío al lograr sus producciones. Así mismo, se debe señalar que hubo preguntas y comentarios de las personas participantes. 
doi: http://dx.doi.org/10.15359/ree.19-3.21

URL: http://www.una.ac.cr/educare

CORREO: educare@una.cr

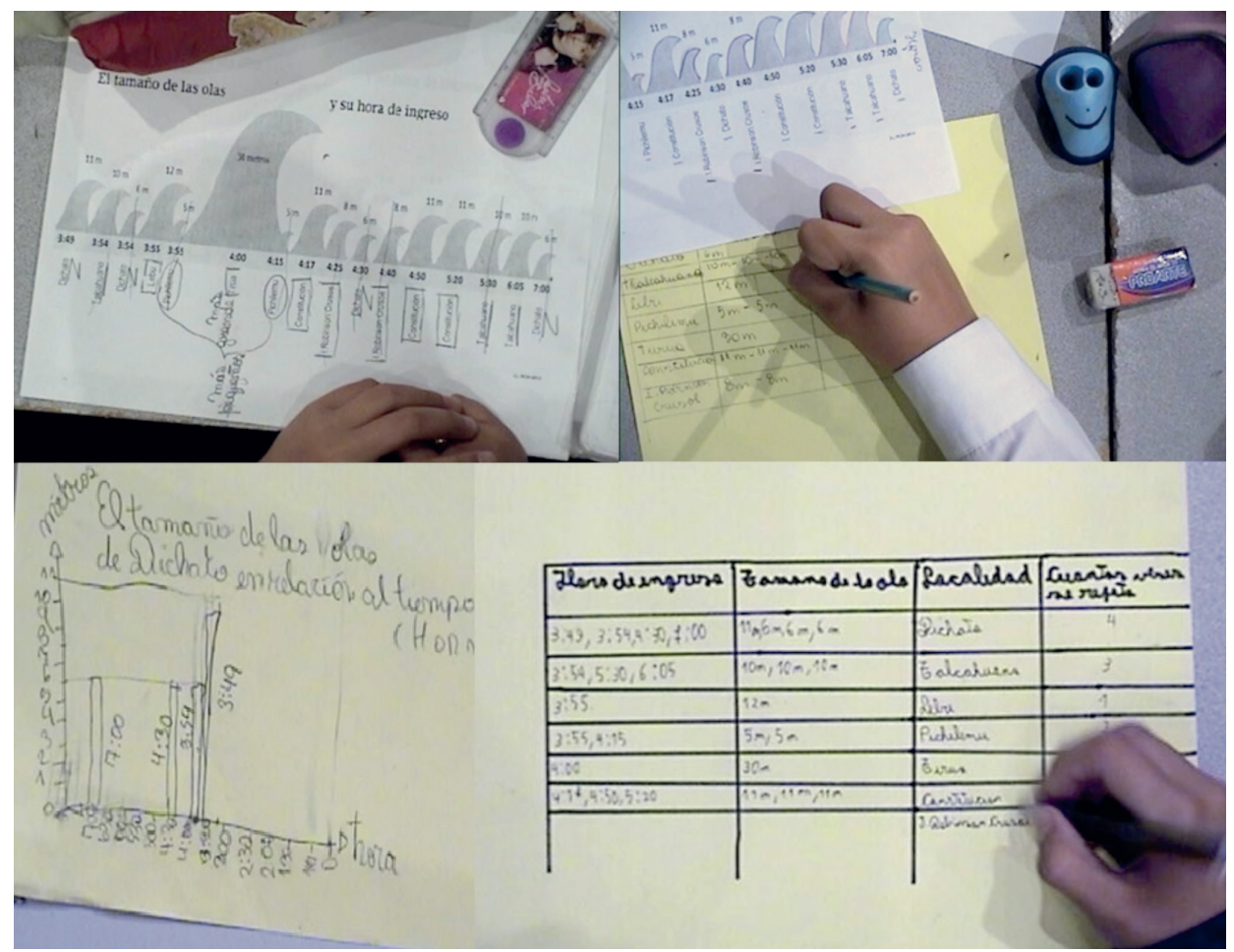

Figura 2. Representaciones de datos de estudiantes: gráficos y tablas.

\section{Instrumento}

Teniendo como antecedente el cuestionario sobre las prácticas y creencias del grupo de docentes de estadística (Zieffler et al., 2012), se crea un instrumento sobre creencias del profesorado en educación estadística. El cuestionario aborda cómo se enseña esta materia, si en una forma más expositiva, centrada en el profesor; o bien en una forma de enseñanza participativa, exploratoria, con interacción grupal, resolviendo problemas y datos en contexto real.

El instrumento utilizado es un conjunto de preguntas que fueron medidas con una escala Likert de cinco puntos, de 1 a 5, (1 es totalmente en desacuerdo y 5 totalmente en acuerdo). Todas las interrogantes fueron evaluadas por un experto en didáctica de la estadística. Se seleccionaron seis ítems claramente relacionados con el objeto de estudio que fuesen afirmaciones simples y comprensibles. Posteriormente, se realizó una aplicación piloto con una muestra de 108 docentes de matemáticas que enseñan estadística, a quienes se les aplicó el instrumento una vez. 
Los datos mostraron simetría con media 2, 2, con un coeficiente de asimetría de Pearson de 0,42 (este coeficiente varía entre -3 y 3 , y cercano a cero la distribución es simétrica). Se obtuvo un "alfa" =0,75. La fiabilidad por ítem, de Gutman, dio valores lambda 0,62, 0,76, 0,74, $0,73,0,75$ y 0,75 para cada uno de los 6 ítems de la escala. Otros estadísticos de fiabilidad son la varianza común de 1,16 , la varianza verdadera $(0,35)$, la varianza de error $(0,81)$ y la fiabilidad de la escala insesgada $(0,73)$. Un análisis factorial exploratorio a tres factores mostró una matriz de covarianza en que los autovalores iniciales eran los mismos en la solución bruta y en la reescalonada. Se asumió un modelo a un factor que explica el 46,5\% del total de la varianza. Con respecto al análisis factorial confirmatorio, el índice de bondad de ajuste fue significativo, $x_{2}^{2}=21,89$ con gl $9(p=0,009)$. Como índice parcial complementario se consideró el cociente $\mathrm{X}^{2}$ /g.l, 2,4, un ajuste razonable (Brooke, Russell y Price, 1988). La media de adecuación muestral de Kaiser-Meyer-Olkin fue 0,69, índice que se usa para aconsejar el análisis factorial. Si KMO > 0,7 indica una alta correlación y, por tanto, conveniencia de AF. En este análisis, la prueba de esfericidad de Barlett fue significativa $(p=0,000)$. Cuanto menor el nivel de significación, más adecuado resulta el análisis factorial.

\section{Procedimiento}

Para el análisis de las respuestas al cuestionario, se aplicó el procedimiento de análisis con base en la prueba Wilcoxon que compara medianas, con la hipótesis estadística "la observación de la clase pública afecta positivamente el cambio en las creencias". Considerando que se estudia un caso de clase pública, y que el propósito es explorar el cambio en las creencias del personal docente, se eligió este estadístico no paramétrico por no cumplir el supuesto de aleatoriedad. A su vez, este estadístico permite reconocer las diferencias en las medianas de las respuestas entre ambas aplicaciones del cuestionario, antes y después de participar en la clase pública.

\section{Resultados}

El promedio para los 25 participantes en el pre test es 2,32; mientras en el post test, es 2,15 (ver figura 3). Los resultados de la investigación muestran que mas allá de la estabilidad de las creencias sobre cómo enseñar estadística para favorecer el aprendizaje, en promedio el grupo de docentes participantes presenta un cambio, a partir de ambas aplicaciones, que no es significativo al 5\% (W=.1). 
doi: http://dx.doi.org/10.15359/ree.19-3.21

URL: http://www.una.ac.cr/educare

CORREO: educare@una.cr

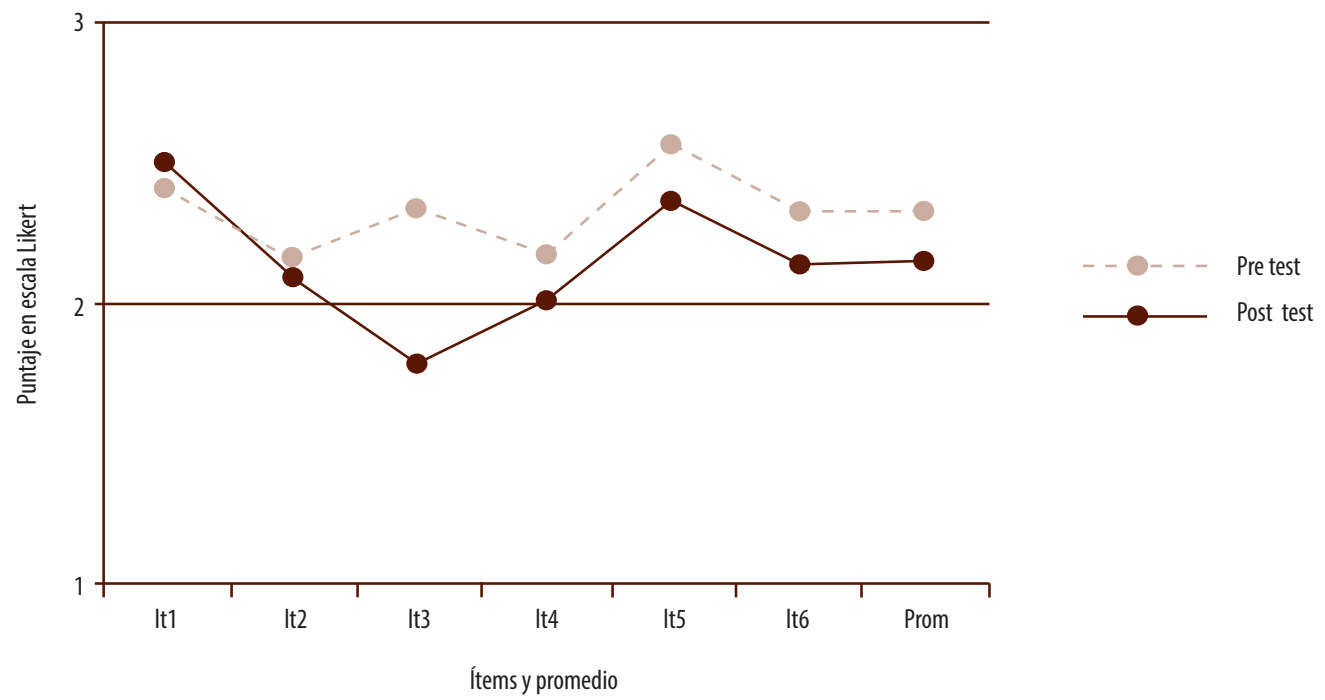

Figura 3. Resultados promedios $(\mathrm{n}=25)$ de cada ítem de la escala, antes y después de la clase pública.

Este resultado muestra que la observación de la clase pública moviliza levemente las creencias sobre "cómo enseñar estadística", pero no de manera significativa. Esta noción atañe a afirmaciones del tipo "Para que los alumnos aprendan Estadística es mejor una clara explicación del profesor que una exploración incierta de los alumnos" y "El ciclo investigativo en Estadística (problema, plan, recogida y análisis de datos, y conclusiones) es más pertinente para la educación secundaria que para la educación primaria" (ver Anexo C).

En todo caso, el leve cambio se moviliza hacia creencias que asocian más los aprendizajes a una forma de enseñanza de carácter participativa, exploratoria, con interacción grupal, resolviendo problemas y datos en contextos reales.

\section{Discusión}

En los antecedentes reportados, las creencias del profesorado determinan las decisiones educativas que realiza en el aula. Estas creencias son difíciles de cambiar, por lo que es uno de los retos de los programas de formación y desarrollo profesional desafiar efectivamente las creencias fundamentales que conducen la toma de decisiones del profesorado (Hart y Carriere, 2011).

Las creencias del grupo de docentes que observaron la clase pública fueron desafiadas en diferentes momentos; inicialmente, al revisar el plan de la lección que se les entregó; luego, al observar la evolución y gestión de la lección; y finalmente al reflexionar junto a los panelistas de la mesa de discusión una vez terminada la clase. 
De este estudio, se considera que la participación en una clase pública de quinto grado modificó, de manera no significativa, las creencias sobre cómo enseñar estadística en la escuela. Esta modificación se vincularía con una forma de hacer clases de tipo participativo, exploratorio, con interacción grupal, resolución de problemas y datos reales.

\section{Conclusiones}

Como se señaló, muchos países han integrado tempranamente la estadística en los currículos escolares de matemática desde los primeros grados. Los programas de estudio promueven desde sus bases que el personal docente desarrolle la alfabetización estadística en sus estudiantes, futuros ciudadanos y ciudadanas de nuestra sociedad. Ello demanda que el profesorado aborde en su enseñanza problemas de interés para el grupo de estudiantes, con datos reales y motivadores; mediante la puesta en juego de situaciones problemáticas que les ayuden a construir libremente argumentos viables y, de esta forma, puedan criticar el razonamiento de otros. Se pretende una enseñanza que entregue oportunidades para combinar la comprensión con los procedimientos con sentido, donde el estudiantado tenga espacio para discutir y encontrar más de una respuesta a una pregunta, y así comience tempranamente a desarrollar su pensamiento estadístico.

La clase pública permitió que el grupo de docentes tuviera la experiencia de percibir y criticar una clase de primaria en tiempo real, con el objetivo de evidenciar el aprendizaje estadístico del estudiantado a través de una clase planificada, en la cual este aprendía conceptos (frecuencia, representaciones estadísticas), utilizaba y creaba herramientas (tablas y gráficos) mediante una exploración libre y con sentido a un problema abierto; una clase en la cual el profesor promovía el trabajo y la discusión en grupos, y un modo de trabajo investigativo propio de un profesional estadístico: problema, plan, análisis de datos y respuesta al problema.

Concretamente, este estudio invita a persistir en mostrar formas de enseñanza realistas, con enfoque abierto y de resolución de problemas, con el fin de afectar las creencias del personal docente, punto crucial para provocar cambios en la enseñanza.

Finalmente, al corresponder a un estudio exploratorio inicial sobre clases públicas de estudio de clases, se generan diferentes proyecciones para continuar investigando. Entre estas, conocer el comportamiento de las diferentes creencias al considerar características de las personas participantes (por ejemplo, años de experiencia, género, entre otros). A su vez, realizar correlaciones entre características de la forma de enseñar la lección (interacción, presentación de desafío, preguntas movilizadoras) y movilización de creencias.

Esta investigación pionera sobre clase pública ofrece la oportunidad de ampliar y poner en análisis las creencias del profesorado; un aspecto fundamental para producir cambios en la educación estadística. La clase pública del estudio de clases es una instancia que permite a personal docente volver a pensar sus prácticas de clases. 
doi: http://dx.doi.org/10.15359/ree.19-3.21

URL: http://www.una.ac.cr/educare

CORREO: educare@una.cr

\section{Referencias}

Araneda, A., del Pino, G., Estrella, S., Icaza, G., y San Martín, E. (2011). Recomendaciones para el currículum escolar del eje Datos y Probabilidad. Recuperado de http://www.soche.cl/ archivos/Recomendaciones.pdf

Ben-Zvi, D. y Sharett-Amir, Y. (2005). How do Primary School Students Begin to Reason about Distributions? [¿Cómo los estudiantes de la Escuela Primaria comienzan a razonar sobre distribuciones?]. En K. Makar (Ed.), Reasoning About Distribution: A Collection of Current Research Studies [Razonamiento sobre Distribución: Una serie de InvestigacionesActuales] [CD-ROM] (pp. 1-27). Brisbane, Australia: University of Queensland.

Brooke, P. P., Rusell, D. W., y Price, J. L. (Mayo, 1988). Discriminant Validation of Measures of Job Satisfaction, Job Involvement, and Organizational Commitment [Validación discriminante de las medidas de satisfacción laboral, participación laboral y compromiso organizacional]. Journal of Aplied Psychology, 73(2), 139-145. doi: http://dx.doi.org/10.1037/00219010.73.2.139

Del Pino, G. y Estrella, S. (2012). Educación estadística: Relaciones con la matemática. Pensamiento Educativo. Revista de Investigación Educacional Latinoamericana, 49(1), 53-64. doi: http:// dx.doi.org/10.7764/PEL.49.1.2012.5

Estrada, A., Batanero, C. y Lancaster, S. (2011). Teachers' Attitudes Towards Statistics [Actitudes de los profesores hacia la Estadística]. En C. Batanero y G. Burrill y C. Reading (Eds.), Teaching Statistics in School Mathematics-Challenges for Teaching and Teacher Education [La enseñanza estadística en los desafíos matemáticos escolares para enseñanza y la educación del profesorado] (pp. 163-174). Netherlands: Springer.

Estrella, S., Olfos, R., y Morales, S. (2014). What Can We Learn from Natural Disasters to Prevent Loss of Life in the Future? [¿Qué podemos aprender de los desastres naturales para evitar pérdida de vidas en el futuro?]. En J. W. Lott y C. J. Lott (Eds.), Lessons Learned from Across the World-PreK-8 [Lecciones aprendidas a través del mundo PreK-8] (pp. 65-70). NCTM, National Council of Teachers of Mathematics. VA: NCTM.

Fang, Y., Lee, C., y Sharifath, B. (2009). Lesson Study in Mathematics: Three Cases from Singapure [Estudio de Clases: tres casos desde Singapure]. En K. Y. Wong, P. Y. Lee, B. Kaur, P. Y. Foong y S. F. Ng (Eds.), Mathematics Education: The Singapure Journey [Educación Matemática: El trayecto de Singapur]. (Series on Mathematics Education: Vol 2., pp. 104-129). Singapure: World Scientific.

Fang, Y., y Lee, C. (2010). Lesson Study and Instructional Improvement in Singapore [Estudio de Clases y la mejora de la enseñanza en Singapur]. (Research Brief No. 10-001). Singapore: National Institution of Singapore. Recuperado de https://www.nie.edu.sg/files/NIE research brief 10 001.pdf 
Felbrich, A., Kaiser, G. y Schmotz, C. (2012). The Cultural Dimension of Beliefs: An Investigation of Future Primary Teachers' Epistemological Beliefs Concerning the Nature of Mathematics in 15 Countries [La dimensión Cultural de las creencias: una investigación de las creencias epistemológicas de futuros profesores de primaria sobre la naturaleza de las matemáticas en 15 países]. ZDM, 44(3), 355-366. doi: http://dx.doi.org/10.1007/s11858-012-0418-x

Fernández, C. y Yoshida, M. (2004). Lesson Study: A Case of a Japanese Approach to Improving Instruction Through School-Based Teacher Development [Estudio de Clase: un caso de un enfoque japonés para mejorar la instrucción a través del desarrollo docente en la escuela]. Mahwah: Lawrence Erlbaum.

Franklin, C. A., y Mewborn, D. S. (2006). The Statistical Education of Grades Pre-K-2 Teachers: A Shared Responsibility [La educación estadística de profesores de grados Pre-K-2: una responsabilidad compartida]. En G. Burrill (Ed.), NCTM 2006 Yearbook: Thinking and Reasoning with Data and Chance [NCTM Anuario 2006: Pensando y razonando con datos y azar] (pp. 335-344). Reston, VA: NCTM.

Fullan, M. G., y Stiegelbauer, S. (1997). El cambio educativo. Guía de planeación para maestros. México: Trillas.

Furtak, E. M., y Alonzo A.C. (2010). The Role of Content in Inquiry-Based Elementary Science Lessons: An Analysis of Teacher Beliefs and Enactment [El rol del contenido en clases de ciencias basadas en la indagación: Un análisis de las creencias del profesor y enactivo]. Research Science Education, 40(3), 425-449. doi: http://dx.doi.org/10.1007/s11165-009-9128-y

Garfield, J., y Ben-Zvi, D. (Diciembre, 2007). How Students Learn Statistics Revisited: A Current Review of Research on Teaching and Learning Statistics [Revisión de Cómo aprenden estadística los estudiantes: Revisión actual de la investigación sobre la enseñanza y el aprendizaje de la estadística]. International Statistical Review, 75(3), 372-396. doi: http:// dx.doi.org/10.1111/j.1751-5823.2007.00029.x

Handal, B. (2003). Teachers' Mathematical Belief: A Review [Creencias matemáticas de los profesores: una revisión]. The Mathematics Educator, 13(2), 47-57 . Recuperado de http:// citeseerx.ist.psu.edu/viewdoc/download?doi=10.1.1.361.2874\&rep=rep1\&type=pdf

Hart, L. C., y Carriere, J. (2011). Developing the Habits of Mind for a Successful Lesson Study Community [Desarrollo de los hábitos de la mente para una comunidad de estudio de clases exitosa]. En L. C. Hart, A. Alston y A. Murata. (Eds.), Lesson Study Research and Practice in Mathematics Education [Estudio de Clases, Investigación y práctica en Educación Matemática] (pp. 27-38). Netherlands: Springer.

Hart, L. C., Alston, A. S., y Murata, A. (Eds.). (2011). Lesson Study Research and Practice in Mathematics Education [Estudio de Clase, Investigación y práctica en Educación Matemática]. Netherlands: Springer. doi: http://dx.doi.org/10.1007/978-90-481-9941-9 
doi: http://dx.doi.org/10.15359/ree.19-3.21

URL: http://www.una.ac.cr/educare

CORREO: educare@una.cr

Hassad, R. A. (2011). Constructivist and Behaviorist Approaches: Development and Initial Evaluation of a Teaching Practice Scale for Introductory Statistics at the College Level Education [Enfoques Constructivista y conductista: Desarrollo y evaluación inicial de una Escala de la Práctica de enseñanza para un curso introductorio de Estadística en la educación de nivel universitario]. Numeracy: Advancing Education in Quantitative Literacy, 4(2), 1-33. doi: http://dx.doi.org/10.5038/1936-4660.4.2.7

Isoda, M., y Olfos, R. (2009). El enfoque de resolución de problemas en la enseñanza de la matemática a partir del estudio de clases. Valparaíso: Ediciones Universitarias de Valparaíso.

Knapp, A., Bomer, M. y Moore, C. (2011). Lesson Study as a Learning Environment for Coaches of Mathematics Teachers Education [Estudio de clase como un ambiente de aprendizaje para los formadores de profesores de matemáticas]. En L. C. Hart, A. Alston y A. Murata. (Eds.), Lesson Study Research and Practice in Mathematics Education Education [Estudio de Clase, Investigación y práctica en Educación Matemática] (pp. 153-164). Netherlands: Springer.

Lewis, C., Perry, R., Hurd, J. y O'Connell, M. P. (Diciembre, 2006). Lesson Study Comes of Age in North America [El Estudio de Clase alcanza la madurez en América del Norte]. Phi Delta Kappan, 88(4), 273-281. Recuperado de http://www.lessonresearch.net/LS 06Kappan.pdf

Ministerio de Educación (MINEDUC). (2012). Matemática. Bases 2012. Santiago de Chile: Autor. Recuperado de http://curriculumenlinea.mineduc.cl/descargar.php?id $\underline{\text { doc }}=201306051003270$

Olfos, R. (Febrero de 2010). Lesson Study in Chile: The Lesson of a Collaboration Program [Estudio de clases en Chile: La clase de un programa de colaboración]. En Fourth APEC - Tsukuba, International Conference: Innovation of Mathematics Teaching and Learning Through Lesson Study -Connection Between Assessment and Subject Matter [En 4ta Conferencia Internacional de APEC, Tsukuba. La innovación en la enseñanza y aprendizaje a travás del Estudio de Clases, Conexión entre evaluación y el contenido disciplinario]. Tokyo, Japan. Recuperado de http:// www.criced.tsukuba.ac.jp/math/apec/apec2009/doc/pdf 20-21/RaimundoOlfos-paper.pdf

Olfos, R., Estrella, S., y Morales, S. (Julio, 2014). Open Lessons Impact Statistics Teaching Teachers'Beliefs [Clases públicas, impacto en las creencias de los profesores en la enseñanza de la estadística]. En K. Makar, B. de Sousa y R. Gould (Eds.), Sustainability in Statistics Education. Proceedings of the Ninth International Conference on Teaching Statistics [Sustentabilidad en Educación Estadística. Actas de la Novena Conferencia Internacional sobre la enseñanza de la estadística] (pp. 1-5). Flagstaff, Arizona, USA. Voorburg. The Netherlands: International Statistical Institute. Recuperado de http://iase-web.org/icots/9/proceedings/pdfs/ICOTS9 10E1 OLFOS.pdf

Pehkonen, E. (2004). State-of-the-art in Mathematical beliefs research [Estado del arte en las creencias matemáticas]. En M. Niss (Ed.), Proceedings of the 10th International Congress on Mathematical Education [Actas del $10^{\circ}$ Congreso Internacional de Educación Matemática]. Roskilde, Denmark: Roskilde University. 
Perry, R. E., y Lewis, C. C. (2009). What is Successful Adaptation of Lesson Study in the US? [¿Qué es una adaptación exitosa de estudio de clases en Estados Unidos?]. Journal of Educational Change, 10(4), 365-391. doi: http://dx.doi.org/10.1007/s10833-008-9069-7

Rock, T. C., y Wilson, C. (2005, invierno). Improving Teaching Through Lesson Study [EI mejoramiento de la enseñanza a través del Estudio de Clases]. Teacher Education Quarterly, 32(1), 77-92. Recuperado de http://www.teqjournal.org/backvols/2005/32 1/ rock\%26wilson.pdf

Shaughnessy, J. M. (2007). Research on Statistics Learning and Reasoning [Investigación sobre aprendizaje y razonamiento estadístico]. En F. K. Lester, Jr. (Ed.), Second Handbook of Research on Mathematics Teaching and Learning [Segundo manual de investigación en enseñanza y aprendizaje de las matemáticas] (pp. 957-1009). Greenwich, CT: Information Age Publishing.

Staub, F. C., y Stern, E. (Junio, 2002). The Nature of Teachers' Pedagogical Content Beliefs Matters for Students' Achievement Gains: Quasi-experimental Evidence from Elementary Mathematics [La naturaleza de las creencias del contenido pedagógico para aumentar el logro de los estudiantes: evidencia cuasi-experimental desde la matemática elemental]. Journal of Educational Psychology, 94(2), 344-355. doi: http://dx.doi.org/10.1037/00220663.94.2.344

Stigler, J. W. y Hiebert, J. (1999). The Teaching Gap [El gap en la enseñanza]. New York, NY: The Free Press.

Yates, S. M. (2006). Elementary Teachers' Mathematics Beliefs and Teaching Practices After a Curriculum Reform [Creencias Matemáticas de profesores de primaria y las prácticas de enseñanza después de una Reforma Curricular]. En J. Novotná, H. Moraová, M. Krátká y N. Stehlíková (Eds.), Proceedings of the $30^{\text {th }}$ Conference of the International Group for the Psychology of Mathematics Education (PME) [Actas de la 30 Conferencia del Grupo Internacional para la Psicología de la Educación Matemática (PME)], 5, 433-440. Recuperado de http://files.eric.ed.gov/fulltext/ED496939.pdf - page=441

Yoong, J. I. (Septiembre-Octubre, 2011). Let Students Tell Us How They Learn [Qué nos dicen los estudiantes acerca de cómo ellos aprenden]. SingTeach, 32, 3-4. Recuperado de http:// singteach.nie.edu.sg/wp-content/uploads/SingTeach Issue32.pdf

Zieffler, A., Park, J., Garfield, J., delMas, R., y Bjornsdottir, A. (2012). The Statistics Teaching Inventory: A Survey on Statistics Teachers' Classroom Practices and Beliefs [El inventario de la enseñanza de la estadística: un estudio sobre las prácticas en salas de clases y las creencias de los profesores de estadística]. Journal of Statistics Education, 20(1), 1-29. Recuperado de http://www.amstat.org/publications/jse/v20n1/zieffler.pdf 


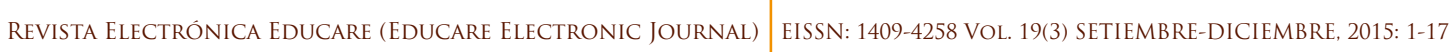

\section{doi: http://dx.doi.org/10.15359/ree.19-3.21}

URL: http://www.una.ac.cr/educare

CORREO: educare@una.cr

Anexo A

Plan de clase

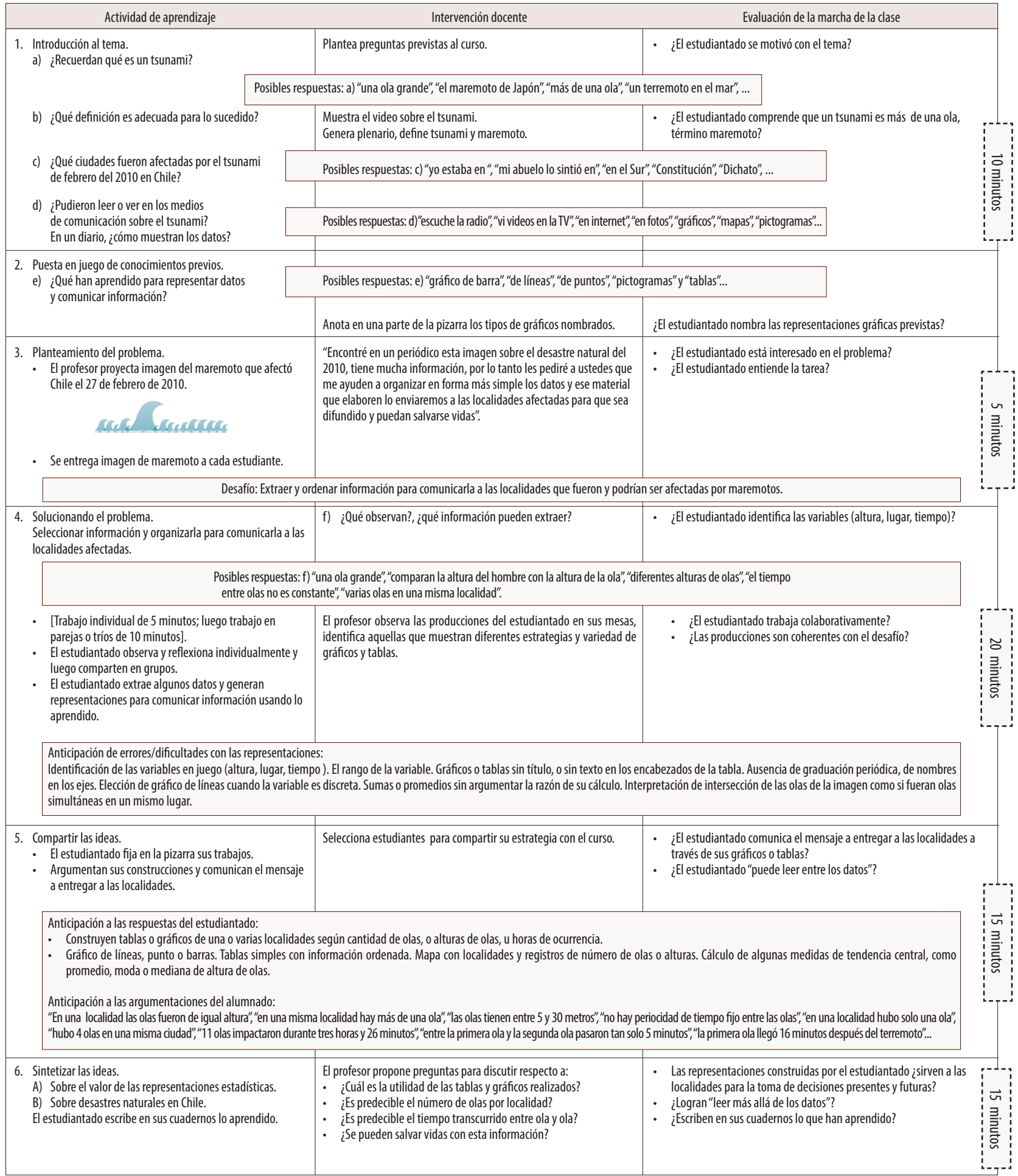




\section{Anexo B}

\section{Lámina entregada a cada estudiante para trabajar en clases}

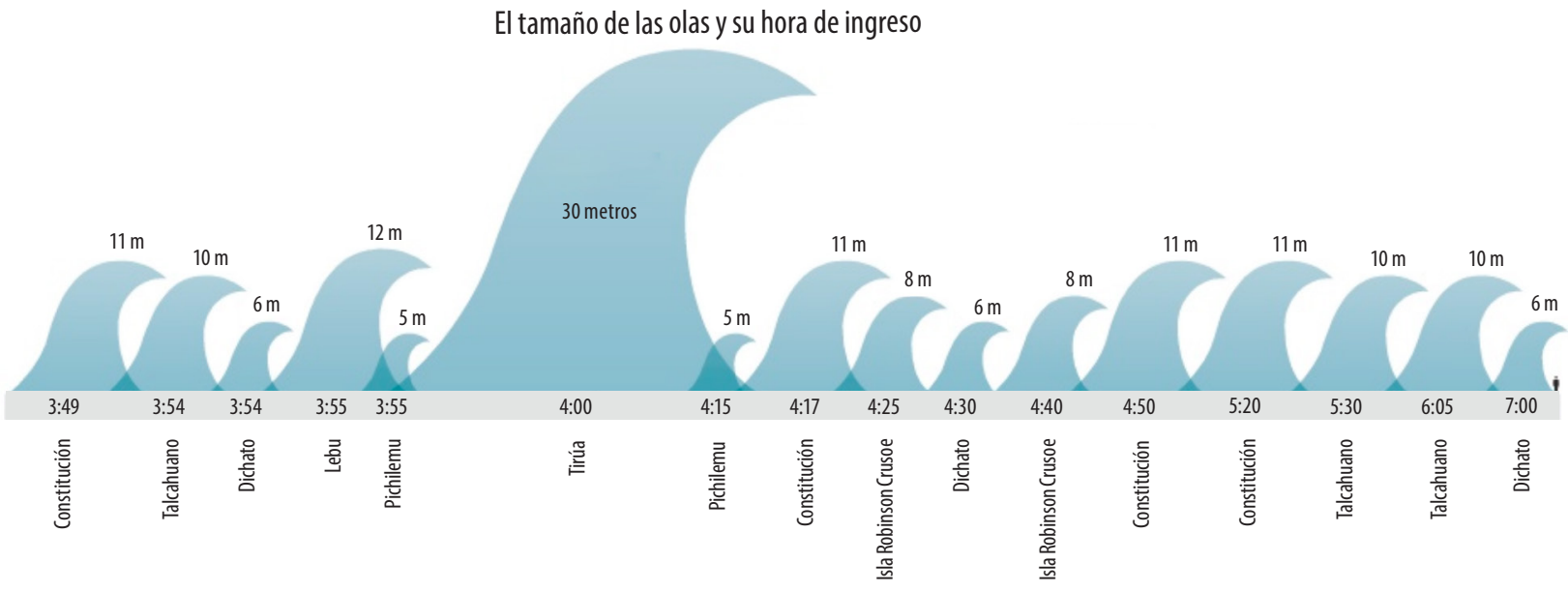

Anexo C

Ítems del cuestionario de creencias

1. Para que el estudiantado aprenda estadística es mejor una clara explicación del profesor que una exploración incierta del grupo de estudiantes.

2. El estudiantado aprende mejor estadística trabajando de manera individual que trabajando en grupo.

3. El ciclo investigativo en estadística (problema, plan, recogida y análisis de datos, y conclusiones) es más pertinente para la educación secundaria que para la educación primaria.

4. Los estudiantes y las alumnas aprenden con efectividad cuando trabajan individualmente y no en grupos.

5. Hay mayor aprendizaje cuando el profesor explica y muestra como se hace una tabla que cuando el alumno o la estudiante observa y explora los datos para una tabla en un contexto real.

6. Los alumnos y las estudiantes aprenden más efectivamente estadística desde una buena exposición del profesor que analizando datos de una situación real.

\section{Cómo citar este artículo en APA:}

Olfos, R., Estrella, S. y Morales, S. (Setiembre-Diciembre, 2015). Clase pública de un estudio de clases de estadística: Una instancia de cambio de creencias en los profesores. Revista Electrónica Educare, 19(3), 1-17. doi: http://dx.doi. org/10.15359/ree.19-3.21

Nota: Para citar este artículo en otros sistemas puede consultar el hipervínculo "Como citar el artículo" en la barra derecha de nuestro sitio web: http://www.revistas.una.ac.cr/index.php/EDUCARE/index 